\title{
O que impede a criatividade? Investigando a influência percebida por estudantes do Ensino Fundamental II de barreiras à criatividade para melhorar a simpatia em relação à criatividade na escola
}

\section{What hinders creativity? Investigating middle school students' perceived influence of barriers to creativity for improving school creativity friendliness}

\author{
Vasiliki Beloyianni* \\ Dimitrios Zbainos*
}

\begin{abstract}
RESUMO
Nos últimos anos, o cultivo da criatividade dos alunos é uma meta amplamente reconhecida da educação moderna. No entanto, várias barreiras impedem a expressão da capacidade criativa dentro do contexto escolar. A influência de muitas barreiras identificadas pela pesquisa pode variar em diferentes contextos culturais, bem como em relação à capacidade criativa individual. A esse respeito, o presente estudo investigou a influência percebida de certas barreiras à criatividade nos alunos do ensino médio grego em termos de gênero e capacidade criativa. Para os propósitos do estudo, 152 jovens adolescentes completaram a versão traduzida do instrumento Barreiras à Criatividade Pessoal (ALENCAR, 1996; MORAIS et al., 2014), enquanto seu potencial criativo foi avaliado pelas escalas gráfico-artísticas do instrumento Avaliação do Potencial Criativo (Evaluation of Creative Potential Battery - EPoC). Os resultados demonstraram que barreiras como
\end{abstract}

*Harokopio University of Athens. Kallithea, Athens, Greece. E-mail: vilbelo@yahoo.gr https://orcid.org/0000-000 - E-mail: zbainos@hua.gr - https://orcid.org/0000-0003-4362-5986 
falta de tempo e oportunidades, inibição, timidez e falta de motivação foram percebidas pelos participantes como obstáculos comuns que afetam consideravelmente sua criatividade, enquanto a repressão social relatou estar afetando sua criatividade em menor grau. Em geral, as percepções de obstáculos à criatividade não pareciam afetar significativamente o potencial criativo ou o gênero dos alunos. Nesse sentido, a alta influência percebida de inibidores específicos na criatividade refletiu um clima nas escolas gregas que, em geral, parece ter pouca simpatia pela criatividade. Assim, as intervenções para aumentar a criatividade dos alunos devem se concentrar na remoção de tais barreiras e na melhoria do clima escolar.

Palavras-chave: Criatividade. Barreiras. Capacidade criativa. Motivação para a criatividade. Pensamento criativo.

\begin{abstract}
In recent years, cultivation of students' creativity is a widely recognized goal of modern education. Yet, various barriers hinder the expression of creative ability within the school context. The influence of many barriers that research has identified may vary within different cultural contexts as well as in relation to individual creative ability. In this regard, the present study investigated the perceived influence of certain barriers to creativity on Greek secondary students in terms of their gender and creative ability. For the purposes of the study, 152 young adolescents completed the translated version of Barriers to Personal Creativity (ALENCAR, 1996; MORAIS et al., 2014), while their creative potential was evaluated by the graphic-artistic scales of the Evaluation of Creative Potential Battery (EPoC). Results demonstrated that barriers such as lack of time and opportunities, inhibition, shyness and lack of motivation were perceived by participants as common obstacles that considerably affect their creativity, whilst social repression was reported to be affecting their creativity to a lesser degree. In general, perceptions of obstacles to creativity did not appear to significantly affect students' creative potential or gender. In this regard, the perceived high influence of specific inhibitors to creativity reflected a climate in Greek schools which in general seems to be of limited friendliness towards creativity. Thus, interventions for enhancing students' creativity should focus on removing such barriers and improving the school climate.
\end{abstract}

Keywords: Creativity. Barriers. Creative ability. Motivation for creativity. Creative thinking. 


\section{Introdução}

A criatividade é considerada uma habilidade fundamental de vida que precisa ser cultivada através da educação para preparar futuros adultos para que possam prosperar em um mundo complexo e incerto (PARKHURST, 1999; SHAHEEN, 2010). Neste sentido, desenvolver o pensamento criativo entre as crianças em idade escolar tem sido uma parte essencial da construção do "capital humano" que determina a "riqueza das nações" (KABANDA, 2014). Nas últimas décadas, as políticas educacionais no mundo inteiro incluíram o desenvolvimento do pensamento criativo entre os objetivos fundamentais da educação formal (BEGHETTO; KAUFMAN, 2014). Em ambientes escolares, várias intervenções educacionais inter e extra-curriculares têm sido propostas para cultivar as habilidades criativas de crianças e adolescentes (COLLARD; LOONEY, 2014; CRAFT, 2005; GRAINGER; BARNES, 2006).

Alguns ambientes pedagógicos e de aprendizagem têm sido sugeridos para facilitar e incentivar a criatividade em um grau mais elevado (CORNER, 2012; JINDAL-SNAPE et al., 2013). Na Grécia, por exemplo, embora o desenvolvimento da criatividade tenha sido reconhecido como um objetivo curricular (PARASKEVOPOULOS, I; PARASKEVOPOULOU, P., 2009), políticas educacionais específicas para a promoção da criatividade quase não têm sido implementadas, e a medida em que o pensamento criativo é promovido e incentivado na prática na sala de aula permanece pouco clara e duvidosa (HAYES, 2004; KAMPYLIS, 2010; PARASKEVOPOULOS, I; PARASKEVOPOULOU, P., 2009; XANTHAKOU, 2012).

Embora nas últimas décadas tenha havido uma quantidade crescente de literatura sobre o desenvolvimento da criatividade em contextos educacionais em todo o mundo, muitas pesquisas têm focado na identificação, compreensão e remoção do que pode ser percebido como inibidor da criatividade de crianças e adolescentes (SADI; AL-DUBAISI, 2008). As pesquisas até agora têm indicado uma variedade de potenciais inibidores da criatividade. Algumas barreiras como autopercepção, motivação, falta de oportunidades e lazer e repressão social têm sido relatadas como sendo as barreiras principais e mais comuns (FILA; PURZER; MATHIS, 2014; HILAL; HUSIN; ZAYED, 2013; KONSTANTINIDOU et al., 2015; MARTIN, 1990; MORAIS; ALMEIDA, 2019). A influência de tais fatores pode explicar em grande medida a variação da criatividade entre e dentro dos indivíduos (AMABILE, 1996, 1998; BEGHETTO, 2010; CREMIN; BURNARD; CRAFT, 2006). 
Em especial, Martin (1990) demonstrou que várias autopercepções, tais como autoconfiança e autoeficácia criativa, podem ser consideradas como barreiras individuais críticas à criatividade. Além disso, pesquisas anteriores indicaram que certos traços de personalidade, tais como timidez, conservadorismo e inibição podem impedir a expressão criativa dos indivíduos num contexto social como a sala de aula (MORAIS et al., 2014; PUCCIO; GRIVAS, 2009). As barreiras individuais têm sido caracterizadas como os principais fortes impedimentos que podem afetar a motivação criativa (CSIKSZENTMIHALYI, 1990; FILA; PURZER; MATHIS, 2014; HILAL; HUSIN; ZAYED, 2013; INTASAO; HAO, 2018; NORDIN; MALIK, 2015).

O papel da motivação intrínseca também tem sido identificado como um fator crítico na criatividade pessoal (AMABILE, 2012; PRABHU; SUTTON; SAUSER, 2008). Em um de seus principais estudos, Amabile (1985) argumentou que a baixa motivação intrínseca tende a ser uma barreira comum entre indivíduos com baixa criatividade. Baixos níveis de motivação intrínseca para a criatividade continuam sendo uma grande "dor de cabeça" para professores e psicólogos educacionais, uma vez que não pode ser adequadamente aprimorada com reforços verbais ou materiais (ALIAKBARI; SADEGHDAGHIGHI, 2013; AMABILE, 1996; FISHER; AMABILE; 2009; PINTRICH; DE GROOT, 1990; VALENZUELA VIANNA; ALENCAR, 2006).

Além das barreiras individuais, vários fatores contextuais têm sido descritos como obstáculos "ambientais" à expressão criativa, tais como falta de recursos, prazos rigorosos, falta de lazer, carga pesada de trabalho e falta de oportunidades de expressão criativa (AMABILE, 1998; CRAFT, 2005; MORAIS et al., 2014; PALETZ, 2012). A repressão social também tem sido relatada como um obstáculo comum à expressão criativa entre os jovens adultos. (ALENCAR, 2001; CRAFT, 2005; MORAIS et al., 2014).

A influência de barreiras específicas à criatividade pode diferir conforme o gênero da pessoa (VERNON, 1989). Por exemplo, Conti, Collins e Picariello (2001) observaram que os meninos em idade escolar tendiam a atingir maior desempenho e maior motivação para a criatividade durante atividades competitivas, enquanto observou-se que as mesmas atividades competitivas reduziam a expressão criativa e o envolvimento das meninas. Por outro lado, a decisão das meninas de agir criativamente parece estar mais relacionada à sua autoestima e autoconceito do que a recompensas externas verbais ou materiais (BAER; KAUFMAN, 2008). A este respeito, supõe-se que homens e mulheres tendem a perceber diferentes fatores como barreiras sérias à sua criatividade (ABRAHAM, 2016; BAER, 1998; BAER; KAUFMAN, 2008; CONTI; COLLINS; PICARIELLO, 2001; MORAIS et al., 2014). 
Identificar e remover barreiras à criatividade pode ser crucial para desenvolver e revelar talentos criativos (GROTH; PETERS, 1999). Pesquisas anteriores demonstram que pessoas altamente criativas tendem a encontrar maneiras de lidar com barreiras como falta de recursos ou ambiguidade (KOZBELT; BEGHETTO; RUNCO, 2010; PLUCKER; MAKEL, 2010; STERNBERG, 2006). Entretanto, o papel mediador da motivação percebida e das barreiras na expressão do potencial criativo individual não tem sido adequadamente estudado.

\section{Objetivos do estudo}

Tem sido argumentado que o conhecimento sobre os fatores que potencialmente prejudicam a criatividade dos jovens estudantes, como descrito por suas autopercepções, beneficiaria o delineamento e a implementação eficaz de intervenções específicas para o desenvolvimento da criatividade (HOFF, 2014; PARAMITHA; INDARTI, 2014). Entretanto, as pesquisas anteriores na área de barreiras à criatividade se preocupavam principalmente com as barreiras percebidas por jovens adultos, e não com as barreiras encontradas por crianças e jovens adolescentes (AMABILE, 1996; NORDIN; MALIK, 2015; SIMONTON, 2000). Além disso, as descobertas relacionadas com as barreiras dos estudantes à criatividade têm se derivado principalmente das percepções e avaliações dos professores (ALIAKBARI; SADEGHDAGHIGHI, 2013; KONSTANTINIDOU et al., 2015) e não a partir das autopercepções das crianças. Especialmente na Grécia, não foi realizado nenhum estudo sobre barreiras à criatividade, nem com adultos nem com crianças.

Assim, este estudo teve como objetivo examinar as barreiras percebidas para a criatividade entre os estudantes dos anos finais do ensino fundamental. Tal investigação forneceria evidências que podem ser utilizadas como ponto de partida para identificar e remover obstáculos na sala de aula, de modo que os alunos possam desenvolver e expressar suas habilidades criativas. Nosso primeiro objetivo era examinar até que ponto os fatores percebidos pelos adultos como barreiras à criatividade tendem a ser reconhecidos como inibidores do pensamento e expressão criativos pelos alunos dos anos finais do ensino fundamental. Outro objetivo do presente estudo foi investigar o efeito de gênero, nível de potencial criativo e escola sobre a influência percebida dessas barreiras específicas para sugerir intervenções apropriadas para promover a criatividade dentro dos ambientes educacionais. 


\section{Método}

\section{Participantes}

A amostra consistia de 152 alunos de 4 escolas, parecidas com escolas do Ensino Fundamental II brasileiro, de vários bairros da região central de Atenas e com idade média de 13,6 anos. Os estudantes na amostra estudavam em todos os três anos do Ensino Fundamental II grego, ( $7^{\circ}$ ano, 16,4\%; $8^{\circ}$ ano, 49,3\%; $9^{\circ}$ ano, $34,2 \%)$, eram de ambos os sexos (52,6\% do sexo feminino) e de origens diferentes (grego 57,2\%, não-grego 42,8\%).

\section{Instrumentos}

Os participantes respondiam a versão traduzida do instrumento Barreiras à Criatividade Pessoal (ALENCAR, 2001), para determinar suas barreiras percebidas à criatividade. $\mathrm{O}$ Barreiras à Criatividade Pessoal é um inventário de autorresposta que contém 44 itens pontuados de acordo com uma escola Likert com cinco pontos (1= "nem um pouco verdadeiro em relação a mim" até $5=$ "muito verdadeiro em relação a mim"). O inventário foi elaborado originalmente no Brasil (ALENCAR, 2001; ALENCAR; FLEITH, 2003), mas tem sido utilizado em outras populações do Sul da Europa para estudar as barreiras percebidas pelos professores e estudantes do ensino superior à criatividade para investigar as oportunidades percebidas para desenvolver e expressar sua criatividade em ambientes educacionais (por exemplo, MORAIS et al., 2014, MORAIS; ALMEIDA, 2019). No presente estudo, foi aplicada uma versão grega adaptada.

Quanto à consistência interna, o alfa do Cronbach foi considerado alto para a amostra principal com $\alpha=0,91$ para todos os 44 itens. Além disso, este instrumento inclui 4 subesclas, a saber: inibição/timidez (15 itens, $\alpha=0,81$ ), falta de motivação (12 itens, $\alpha=0,73$ ), falta de tempo/oportunidades ( 9 itens, $\alpha=0,72)$ e repressão social ( 8 itens, $\alpha=0,61$ ).

A fim de avaliar a capacidade criativa, aplicou-se a escala gráfica do instrumento Avaliação do Potencial Criativo (Evaluation of Creative Potential Battery - EPoC) (LUBART; ZENASNI; BARBOT, 2013). O EPoC é uma ferramenta de medição atualizada da criatividade de crianças e jovens adolescentes. A escala gráfica deste instrumento examina os dois principais processos do pensamento criativo, o pensamento convergente e divergente, tanto integrativo como exploratório dentro de quatro desafios diferentes de expressão gráfica criativa. $\mathrm{O}$ EPoC gráfico tem sido descrito como um instrumento que não sofre enviesamento por influência cultural (LUBART; ZENASNI; BARBOT, 
2013). Os dois testes do pensamento divergente são avaliados a partir de uma escala Likert com 7 pontos de acordo com o número de desenhos diferentes onde $1=$ " 0 ou 1 desenho" e 7 = "14 ou mais desenhos". Os dois testes do pensamento convergente são avaliados a partir de critérios de qualidade de originalidade e adaptação de acordo com uma escala Likert com 7 pontos ( 1 = "muito fraco/ ausência completa de ideias" a 7 = "ideia muito original").

\section{Procedimentos}

A realização da pesquisa foi aprovada pelo Ministério da Educação da Grécia (Código de Autorização: 61407/D2/12-04-2016) a partir da proposta do Instituto de Políticas Educacionais que examinou as questões éticas e a metodologia. Posteriormente, uma carta foi enviada aos pais dos alunos, incluindo informações detalhadas sobre o objetivo do estudo, o método de coleta de dados e a garantia de anonimato e confidencialidade. Foi obtido o consentimento por escrito dos pais de todos os alunos envolvidos.

Os participantes realizaram as tarefas dadas trabalhando individualmente na sala de aula sob a supervisão do pesquisador dentro do espaço de uma hora. Os primeiros cinco minutos foram utilizados para explicações sobre o processo e exigências da pesquisa. Depois, os participantes tiveram dez minutos para responder uma versão traduzida do inventário de Barreiras à Criatividade Pessoal e os demais 40 minutos para realizar as tarefas do EPoC.

\section{Resultados}

Inicialmente, a foi aplicada estatística descritiva para fornecer informações sobre a influência percebida pelos estudantes das barreiras comuns à criatividade. Em geral, a amostra relatou "falta de tempo e oportunidades $(M=3,32, D P=0,65)$ e "inibição ou timidez" $(M=3,10, D P=0,64)$ como os dois obstáculos mais predominantes à sua criatividade, seguidos por "falta de motivação" $(M=3,07$, $D P=0,60)$ e "repressão social" $(M=3,07, D P=0,60)$. Aqui, os estudantes gregos dos anos finais do ensino fundamental apontaram tanto fatores internos quanto sociais como barreiras frequentes para seu pensamento e atuação criativos.

Medidas repetidas com base na análise ANOVA mostraram um efeito principal estatisticamente significativo dos diferentes tipos de barreiras à criatividade $\operatorname{com} F(3,148)=55,80, p<0,05$, parcial $\eta 2=0,53$. Para investigar a fonte deste efeito significativo, um teste de Bonferroni para comparações post 
hoc demonstrou que as barreiras relevantes para "falta de motivação", "falta de tempo/oportunidades" e "repressão social" diferiram significativamente uma da outra no nível de significância de 0,05 . As barreiras relacionadas à "inibição/ timidez" diferiram significativamente de "falta de tempo/oportunidades", e "repressão social" $(p<0,05)$. Entretanto, não foram encontradas diferenças estatisticamente significativas entre "inibição/timidez" e "falta de motivação" ( $p$ $>0,05)$. O achado acima implica que as barreiras examinadas não são percebidas como igualmente importantes, exceto no que diz respeito à "inibição e timidez".

$\mathrm{A}$ análise das respostas dos participantes às tarefas gráficas do $\mathrm{EPoC}$ demonstrou que a amostra tendia a exibir um potencial criativo baixo a moderado no total, levando em conta a escala de pontuação de 7 pontos. Como visto na Tabela 1, foram encontradas pontuações mais altas em testes de "pensamento convergante exploratório criativo" e "pensamento convergente integrative criativo" do que em tarefas relacionadas a "pensamento divergente criativo", o que pode indicar uma tendência de conformidade.

TABELA 1 - DESCRITORES DA HABILIDADE CRIATIVA DOS ESTUDANTES $(\mathrm{N}=152)$

\begin{tabular}{|l|c|c|}
\hline & $M(D P)$ & $C I 95 \%$ \\
\hline Pensamento divergente exploratório (forma abstrata) & $1,93^{\mathrm{a}}(1,02)$ & {$\left[\begin{array}{ll}1,76 & 2,09\end{array}\right]$} \\
\hline Pensamennto divergente integrativo (forma concreta) & $1,90^{\mathrm{b}}(1,03)$ & {$\left[\begin{array}{ll}1,74 & 2,06\end{array}\right]$} \\
\hline Pensamento convergente exploratório (formas abstratas) & $3,60^{\mathrm{ab}}(1,87)$ & {$\left[\begin{array}{ll}3,30 & 3,90\end{array}\right]$} \\
\hline Pensamento convergente integrativo (formas concretas) & $3,15^{\mathrm{ab}}(1,61)$ & {$\left[\begin{array}{ll}2,90 & 3,41\end{array}\right]$} \\
\hline Criatividade Total & $2,64(0,89)$ & {$\left[\begin{array}{ll}2,50 & 2,79\end{array}\right]$} \\
\hline
\end{tabular}

Nota: As médias com os mesmos superíndices são significativamente diferentes umas das outras. FONTE: Elaborado pelos autores.

Medidas repetidas com base na análise ANOVA revelaram que o efeito principal dos diferentes tipos de pensamento criativo era estatisticamente significativo, $F(3,148)=39,66, p<0,05, \eta 2=0,45$. Foi realizada uma análise post hoc de Bonferroni para investigar a fonte deste efeito significativo. A análise mostrou que os valores médios para "pensamento divergente integrativo", "pensamento convergente exploratório" e "pensamento convergente integrativo" diferiam significativamente um do outro $(\mathrm{p}<0,05)$. $\mathrm{O}$ "pensamento divergente exploratório" diferiu significativamente das outras variáveis, exceto o "pensamento divergente integrativo" $(p>0,05)$.

Para examinar o efeito do gênero nas barreiras percebidas pelos estudantes à criatividade, foi realizada uma série de análises de variância (ANOVA). Os resultados mostraram que a "falta de motivação" como uma barreira percebida 
à criatividade foi significativamente afetada pelo gênero $(F(1,150)=6,86, \mathrm{p}$ $<0,05, \eta 2=0,044)$. Aparentemente, os estudantes masculinos perceberam a "falta de motivação" mais frequentemente como um obstáculo à sua criatividade pessoal $(M=3,21, D P=0,58)$ do que seus pares femininos $(M=2,95, D P=$ $0,60)$. Não foram encontrados efeitos estatisticamente significativos de gênero para as outras barreiras percebidas. Assim, o contexto cultural e escolar específico parecia ser menos desafiador e estimulante para a criatividade dos meninos.

Para investigar o efeito de contextos escolares específicos sobre a influência percebida pelos estudantes das barreiras à criatividade acima mencionadas, foi realizada uma série de análises de variância (ANOVA). Os resultados mostraram que as percepções dos alunos sobre as barreiras específicas à criatividade não diferiram significativamente de escola para escola. Portanto, poderia-se supor que essas quatro escolas se caracterizássem por um clima criativo semelhante.

Para investigar o possível efeito da capacidade criativa sobre a influência percebida pelos estudantes das barreiras comuns à sua criatividade, a amostra foi dividida em três grupos, de acordo com os níveis de criatividade dos estudantes. O "grupo de baixa criatividade" consistia de estudantes com os $20 \%$ mais baixos do total de pontos nas tarefas de criatividade do EPoC e o total de pontos de 4 a 7. Respectivamente, o "grupo de alta criatividade" consistia de estudantes com os $20 \%$ mais altos do total de pontos nas tarefas do EPoC, com nota 14 ou acima. Os demais alunos, com pontuação total entre 8 e 13, foram classificados como o "grupo de criatividade média". Todos os três grupos diferiram significativamente entre si em termos de sua capacidade criativa $(F(2,149)=321,04, p<0,001)$.

Uma série de análises de variância (ANOVA) mostrou que não houve efeito estatisticamente significativo das pontuações dos estudantes no EPOC sobre sua influência percebida em qualquer uma das barreiras à criatividade examinadas. Esta constatação pode confirmar que existe um clima comum entre as quatro escolas examinadas, que é percebido como menos favorável à criatividade pelos alunos, independentemente de seu nível de potencial criativo.

\section{Discussão}

Como mencionado acima, tanto a autopercepção da criatividade quanto as percepções sobre a simpatia do contexto para com a criatividade podem afetar até certo ponto a decisão dos indivíduos de pensar e agir criativamente (ALENCAR; FLEITH; MARTINEZ, 2011; ELISONDO, 2016; ZIEGLER, 2012). O valor da identificação e compreensão de tais percepções não é apenas teórico, mas 
também prático, pois pode levar a uma intervenção direcionada e eficaz para aumentar a criatividade dentro da sala de aula (COLLARD; LOONEY, 2014). A este respeito, este estudo procurou contribuir para as poucas pesquisas existentes a respeito da influência percebida de barreiras específicas à criatividade, concentrando-se em estudantes dos anos finais do ensino fundamental na Grécia.

Os achados do presente estudo estão em consonância com pesquisas anteriores que demonstraram a insuficiência de recursos, a falta de motivação e a timidez como as barreiras mais fortes percebidas para o pensamento e a expressão criativos, enquanto a repressão social tem sido referida como um obstáculo menos frequente (ALENCAR, 2001; ALENCAR; FLEITH; MARTINEZ, 2011).

"Falta de tempo e oportunidades" para a expressão criativa, foi relatada como a barreira mais predominante que desincentiva os estudantes dos anos finais do ensino fundamental na Grécia a pensarem e agirem de forma criativa. Este achado pode ser interpretado através da perspectiva mais ampla dos achados de pesquisas anteriores que mostraram que o dinamismo criativo dos currículos pode ser sufocado e que as oportunidades de expressão criativa podem ser restringidas nas escolas orientadas para provas e avaliações padronizadas com base em critérios acadêmicos (DAVIS, 2013; ROBINSON; ARONICA, 2016). Uma cultura escolar performativa para atingir um nível acadêmico rigoroso tem sido descrita como conflitante com abordagens criativas de pensamento e aprendizagem (CORNER, 2012).

As escolas gregas parecem ter todas as características de um contexto educacional que não é simpático à criatividade, o que leva a uma falta de tempo e de oportunidades para a criatividade. Primeiramente, os currículos gregos não fornecem uma definição precisa de criatividade nem diretrizes específicas para desenvolver a criatividade na sala de aula. Cachia, Ferrari e Punie (2010) em seu estudo nos países membros da União Europeia demonstraram que a falta de uma definição clara de criatividade e de ferramentas educacionais apropriadas nos currículos levou a oportunidades limitadas de criatividade dentro do contexto escolar em vários países europeus, incluindo a Grécia. Os currículos gregos têm sido relatados pelos educadores como sendo inflexíveis e rigorosos e, portanto, um importante obstáculo para promover a criatividade dos estudantes (KONSTANTINIDOU et al., 2015). Além dos currículos, os professores gregos têm conhecimento limitado das ferramentas e técnicas apropriadas para identificar e promover a criatividade na sala de aula (KAMPYLIS, 2010). A falta de tempo e os trabalhos de casa pesados parecem ser também uma característica atual da escola de Ensino Fundamental II na Grécia. Segundo a OECD (2011), o sistema educacional grego está entre aqueles que exigem que os alunos passem uma grande quantidade de tempo semanal em aulas pós-escolares de matemática, ciências e idiomas. Assim, a prevalência da falta de tempo e oportunidade como 
uma barreira à criatividade dos alunos do ensino médio grego não é um achado surpreendente.

"Inibição", "timidez" e "falta de motivação" foram relatados em ordem de importância como barreiras enfrentadas pelos estudantes secundários gregos. Estas também foram frequentemente reconhecidas anteriormente como barreiras aos esforços de criatividade na literatura (ALENCAR, 2001; ALENCAR; FLEITH; MARTINEZ, 2011; BANAJI; CRANMER; PERROTTA, 2013; KONSTANTINIDOU et al., 2015; MORAIS et al., 2014). Os estudantes tendem a se sentir tímidos e inibidos para expressar suas ideias criativas se souberem que as ideias criativas não seriam compreendidas e apreciadas pelos professores. Tem sido relatado que a criatividade tende a ser restringida nas escolas secundárias gregas (PARASKEVOPOULOS, I; PARASKEVOPOULOU, P., 2009) e que os estudantes estão sendo educados e avaliados majoritariamente por fatores outros que sua criatividade (ZBAINOS; BELOGIANNI, 2018). A este respeito, os estudantes raramente são desafiados a expressar ideias originais e inovadoras, já que a criatividade parece ser menos valorizada ou recompensada na sala de aula, resultando em baixa motivação. Ainda, as escolas podem aumentar a inibição e a timidez, pois a expressão espontânea da ideação criativa pode ser percebida pelos professores como um comportamento desviante que precisa ser restrito para que o cumprimento do padrão desejado na sala de aula seja facilitado (PARASKEVOPOULOS, I; PARASKEVOPOULOU, P., 2009), para que as metas acadêmicas dos currículos possam ser atingidas de forma eficiente e oportuna (KONSTANTINIDOU et al., 2015). Portanto, para reforçar a motivação para a criatividade e melhorar a expressão criativa, as escolas devem promover a abertura aos estímulos culturais, o interesse em diferentes pontos de vista, incentivos e recompensas (DUBINA; RAMOS, 2013).

No entanto, surpreendentemente, apesar do contexto educacional com as características mencionadas acima, os estudantes gregos não pareciam dar tanta importância à "repressão social" quanto aos obstáculos à criatividade acima mencionados. Eles relataram que a influência de fatores relacionados à repressão social, como o reconhecimento social limitado e a recompensa da capacidade criativa, têm um efeito negativo na expressão da criatividade, ainda que em menor grau em comparação com os outros tipos de barreiras examinados. Este achado pode implicar que os estudantes tendem a perceber o clima escolar das escolas secundárias gregas como indiferente à criatividade, onde o pensamento criativo e a expressão inovadora não são abertamente oprimidos ou recompensados. Pesquisas anteriores realizadas com adultos também demonstraram que a repressão social era percebida como um obstáculo presente, mas menos importante, aos comportamentos criativos (ALENCAR, 2001; ALENCAR; FLEITH, 2003; CRAFT, 2005; MORAIS et al., 2014). 
Com relação ao efeito do gênero sobre as barreiras percebidas à criatividade, o presente estudo não encontrou muita variação significativa. A única diferença significativa entre os dois gêneros estava relacionada à "falta de motivação", que foi percebida como um obstáculo mais forte à criatividade dos meninos do que a das meninas. Este achado está de acordo com pesquisas anteriores que encontraram alguma variação nas percepções de homens e mulheres relacionadas ao estímulo motivacional para a criatividade (BAER, 1998; BAER; KAUFMAN, 2008; CONTI; COLLINS; PICARIELLO, 2001; MORAIS et al., 2014). Por exemplo, a expectativa de um feedback e avaliação não quantificados foi relatada como tendo um impacto positivo na motivação para a criatividade dos adolescentes masculinos, mas parecia diminuir a motivação de seus pares femininos, implicando uma peculiar "psicologia social da criatividade conforme o gênero" (BAER, 1997, 1998).

Por último, no que diz respeito aos processos cognitivos medidos pelo EPoC, as pontuações para o pensamento divergente pareciam ser extremamente baixas e significativamente inferiores às pontuações para o pensamento convergente criativo. Este achado pode ser interpretado pelo fato de que os estudantes gregos não estão familiarizados com tarefas que exigem fluência ideacional criativa que é cultivada em contextos que promovem a assunção de riscos, inconformismo e curiosidade. Além disso, neste estudo, descobriu-se que a influência percebida de inibidores específicos não foi significativamente afetada pelo nível de potencial criativo dos estudantes. Este achado foi bastante surpreendente, uma vez que a literatura anterior indicou que várias barreiras individuais e ambientais à criatividade podem afetar tanto o pensamento criativo quanto a expressão criativa (AMABILE, 1996; COLLARD; LOONEY, 2014; HENNESSEY; AMABILE, 2010). Parece que nas escolas na nossa amostra predomina um clima generalizado de simpatia limitada em relação à criatividade que afeta a maioria dos indivíduos em toda a gama de habilidades criativas (HUNTER; BEDELL; MUMFORD, 2007).

\section{Recomendações educacionais e implicações práticas}

Para concluir, examinar a influência percebida de vários fatores dentro de uma abordagem sistêmica da criatividade poderia contribuir para a tentativa não apenas de implementar intervenções eficientes para o desenvolvimento da criatividade dentro de ambientes educacionais, mas também de formar mais actiotopos (actiotopes) favoráveis à criatividade para os indivíduos. Como os actiotopos favoráveis à criatividade constituem construtos subjetivos, levar em 
conta as necessidades e percepções pessoais dos estudantes continua sendo crítico (FLEITH, 2011; ZIEGLER, 2012; ZIEGLER; VIALLE, 2009). Para promover originalidade, novidade e pensamento criativo, as escolas modernas devem ser transformadas em contextos onde a criatividade é valorizada e explorada dentro de currículos flexíveis, com a remoção de inibidores potenciais. A construção de um clima de apoio à criatividade é essencial para estimular e incentivar atitudes criativas na escola (PETER-SZARKA, 2012) e é nisto que as escolas gregas devem focar em superar barreiras e permitir que a criatividade dos estudantes seja expressa.

Para melhorar a simpatia para com a criatividade escolar, as políticas educacionais centrais devem estabelecer e oferecer as circunstâncias apropriadas. A este respeito, uma definição ampla e clara de criatividade e uma orientação apropriada sobre como alimentá-la deve ser fornecida nos currículos. Além disso, as políticas educacionais devem ser revistas e revisadas regularmente para promover as necessidades mutáveis de aprendizagem das sociedades modernas e facilitar o desenvolvimento das competências-chave do século 21. As mudanças no currículo devem ser seguidas por mais reformas na cultura educacional central e nos princípios de avaliação educacional para permitir flexibilidade no ensino e, também, valorizar e premiar o pensamento criativo.

$\mathrm{O}$ treinamento de professores para poder identificar, avaliar e promover a criatividade na sala de aula é parte essencial da construção de escolas que sejam simpáticas para com a criatividade. Embora o desenvolvimento da criatividade seja um dos objetivos de aprendizado na escola secundária grega, não há diretrizes sugeridas ou ferramentas de ensino para ajudar os professores a implementá-la na prática. Os currículos devem ser acompanhados de ferramentas de orientação para guiar a intervenção dos professores a fim de desenvolver e incentivar a criatividade. Além disso, programas de treinamento de professores devem ser aplicados para fornecer orientação personalizada, experiências de ensino criativas e oportunidades para compreender e valorizar a criatividade. Os achados do presente estudo podem contribuir para esforços voltados para o desenvolvimento de contextos favoráveis à criatividade para os estudantes, onde intervenções eficientes para a criatividade podem ocorrer. Como os ambientes favoráveis à criatividade constituem construtos subjetivos, é essencial levar em conta as necessidades pessoais dos alunos e suas percepções sobre suas barreiras à criatividade (FLEITH, 2011; ZIEGLER, 2012; ZIEGLER; VIALLE, 2009). Portanto, as escolas no mundo inteiro em geral, mas em particular as escolas gregas, devem se focar em como remover as barreiras percebidas através da construção de um clima de apoio à criatividade é essencial para estimular e incentivar atitudes criativas na escola (PETER-SZARKA, 2012) para promover originalidade, novidade e pensamento criativo. 


\section{Limitações e indicações para pesquisas futuras}

O presente estudo investigou a influência percebida de fatores específicos como barreiras à criatividade entre os estudantes dos anos finais do ensino fundamental, um tópico que não foi examinado anteriormente em relação à população grega. Entretanto, o pequeno tamanho da amostra restringe a medida em que os resultados podem ser generalizados para a população de adolescentes gregos como um todo. Para compreender plenamente o papel de elementos específicos dos ambientes percebidos em relação à criatividade, é necessária uma investigação mais aprofundada com amostras maiores.

Além disso, os instrumentos de autorrelato geralmente não revelam outros fatores latentes que podem afetar a capacidade ou decisão de um indivíduo de realizar seu potencial (PAULHUS; VAZIRE, 2007), portanto, pesquisas futuras devem aplicar vários métodos, tanto quantitativos como qualitativos, para investigar o impacto de vários fatores como inibidores da criatividade que podem não ser privados ou observados pelos métodos de auto-relato.

Além disso, os instrumentos de autorrelato geralmente não revelam outros fatores latentes que podem afetar a capacidade ou decisão de um indivíduo de realizar seu potencial (PAULHUS; VAZIRE, 2007), portanto, pesquisas futuras devem aplicar vários métodos, tanto quantitativos como qualitativos, para investigar o impacto de vários fatores enquanto inibidores da criatividade que podem não ser derivados ou observados pelos métodos de autorrelato.

Outra limitação é que as tarefas do EPoC, utilizadas para avaliar a criatividade dos estudantes, não foram padronizadas para a população grega para garantir a validade e a confiabilidade dos dados coletados. Pesquisas futuras precisam utilizar uma combinação de instrumentos apropriados e padronizados para a população grega.

\section{REFERÊNCIAS}

ABRAHAM, Anna. Gender and creativity: an overview of psychological and neuroscientific literature. Brain Imaging and Behavior, Bethesda, v. 10, n. 2, p. 609618, 2016.

ALENCAR, Eunice M. L. Barreiras à criatividade pessoal: desenvolvimento de um instrumento de medida. In: REUNIÃO ANUAL DE PSICOLOGIA, 26., 1996, Ribeirão 
Preto. Anais [...]. Ribeirão Preto: SBP, 1996. Disponível em: https://pdfs.semanticscholar. org/30b8/66f9330981cb7c238ff6eeb5f292270295e0.pdf. Acesso em: 30 out. 2021.

ALENCAR, Eunice M. L. Obstacles to Personal Creativity among University Students. Gifted Education International, New York, v. 15, n. 2, p. 133-140, 2001.

ALENCAR, Eunice M. L.; FLEITH, Denise. Barreiras à criatividade pessoal entre professores de distintos níveis de ensino. Psicologia: Reflexão e Crítica, Porto Alegre, v. 16, n. 1, p. 63-69, 2003. Disponível em: https://www.scielo.br/j/prc/a/MhfntvD8D9V XsMYvQxdXDpm/?lang=pt. Acesso em: 30 out. 2021.

ALENCAR, Eunice M. L.; FLEITH, Denise; MARTINEZ, Albertina. Obstacles to Personal Creativity between Brazilian and Mexican University Students: A Comparative Study. The Journal of Creative Behavior, [S. l.], v. 37, n. 3, p. 179-192, 2011.

ALIAKBARI, Mohammad; SADEGHDAGHIGHI, Akram. Teachers' Perception of the Barriers to Critical Thinking. Procedia - Social and Behavioral Sciences, [S. l.], n.70, p. 1-5, 2013.

AMABILE, Teresa M. Motivation and creativity: Effects of motivational orientation on creative writers. Journal of Personality and Social Psychology, [S. l.], v. 48, n. 2, p. 393-399, 1985. Disponível em: https://psycnet.apa.org/record/1985-19751-001. Acesso em: 30 out. 2021.

AMABILE, Teresa M. Creativity in context. Boulder, CO: Westview Press, 1996.

AMABILE, Teresa M. How to Kill Creativity. Harvard Business Review, [S. l.], v. 76, n. 5, p. 76-87, 1998.

AMABILE, Teresa M. Componential Theory of Creativity. Harvard Business School Working Paper, [S. l.], p. 12-96, 2012.

BAER, John. Gender differences in the effects of anticipated evaluation on creativity. Creativity Research Journal, [S. l.], v. 10, n. 1, p. 25-31, 1997.

BAER, John. Gender Differences in the Effects of Extrinsic Motivation on Creativity. The Journal of Creative Behavior, [S. l.], v. 32, n. 1, p. 18-37, 1998.

BAER, John; KAUFMAN, James C. Gender Differences in Creativity. The Journal of Creative Behavior, [S. l.], v. 42, n. 2, p. 75-105, 2008.

BANAJI, Sakuntala; CRANMER, Sue; PERROTTA, Carlo. What's stopping us? Barriers to creativity and innovation in schooling across Europe. In: THOMAS, Kerry; CHAN, Janet (ed.). Handbook of Research on Creativity. Cheltenham, UK: Edward Elgar Publishing, 2013. p. 450-463.

BEGHETTO, Ronald. Creativity in the classroom. In: STERNBERG, Robert J.; KAUFMAN, John C. (ed.). The Cambridge handbook of creativity. New York NY, US: Cambridge University Press, 2010. p. 447-463. 
BEGHETTO, Ronald; KAUFMAN, James C. Classroom contexts for creativity. High Ability Studies, [S. l.], v. 25, n. 1, p. 53-69, 2014.

CACHIA, Romina; FERRARI, Anusca; PUNIE, Yves. Creative Learning and Innovative Teaching. Final Report on the Study on Creativity and Innovation in Education in the EU Member States. Luxembourg: JRC-Publications Office of the European Union, 2010.

COLLARD, Paul; LOONEY, Janet. Nurturing Creativity in Education. European Journal of Education, [S. l.], v. 49, n. 3, p. 348-364, 2014.

CONTI, Regina; COLLINS, Mary Ann; PICARIELLO, Martha L. The impact of competition on intrinsic motivation and creativity: considering gender, gender segregation and gender role orientation. Personality and Individual Differences, [S. l.], v. 31, n. 8, p. 1273-1289, 2001.

CORNER, Claire. Into another world: From creativity to creative learning. Improving Schools, [S. l.], v. 15, n. 2, p. 116-129, 2012.

CRAFT, Anna. Creativity in Schools: Tensions and Dilemmas. London: Routledge, 2005.

CREMIN, Teresa; BURNARD, Pamela; CRAFT, Anna. Pedagogy and possibility thinking in the early years. International Journal of Thinking Skills and Creativity, [S. l.], v. 1, n. 2, p. 108-119, 2006.

CSIKSZENTMIHALYI, Mihaly. The domain of creativity. In: RUNCO, Mark Andrew; ALBERT, Robert S. (ed.). Theories of creativity. CA: Sage Publications, Inc, 1990. v. 115, p. 190-212.

DAVIS, John M. Supporting creativity, inclusion, and collaborative multi-professional learning. Improving Schools, [S. l.], v. 16, n. 1, p. 5-20, 2013.

DUBINA, Igor N.; RAMOS, Suzanna J. Creativity Across Cultures. In: CARAYANNIS, Elias (ed.). Encyclopedia of Creativity, Invention, Innovation and Entrepreneurship. New York, NY, US: Springer New York, 2013. p. 360-364.

ELISONDO, Romina. Creativity is Always a Social Process. Creativity. Theories Research-Applications, [S. l.], v. 3, n. 2, p. 194-210, 2016.

EYSENCK, Hans J. The roots of creativity: Cognitive ability or personality trait? Roeper Review, [S. l.], n. 5, n. 4, p. 10-12, 1983.

FILA, Nicholas; PURZER, Senay; MATHIS, Paul D. I'm not the creative type: Barriers to student creativity within engineering innovation projects. In: AMERICAN SOCIETY FOR ENGINEERING EDUCATION- ASEE ANNUAL CONFERENCE \& EXPOSITION, 121 $1^{\text {st }}$, 2014, Indianapolis. Paper [...]. Indianapolis: ASEE, 2014. Disponível em: https://monolith.asee.org/public/conferences/32/papers/9602/view. Acesso em: 05 maio 2021.

FISHER, Colin; AMABILE, Teresa M. Creativity, improvisation and organizations. In: RICKARDS, Tudor; RUNCO, Mark Andrew et al. (ed.). The Routledge Companion to Creativity. New York: Routledge, 2009. p. 13-24. 
FLEITH, David S. Creativity in the Brazilian Culture. Online Readings in Psychology and Culture, [S. l.], v. 4, n. 3, 2011.

GRAINGER, Teresa; BARNES Jonathan. Creativity in the Primary School Curriculum. In: ARTHUR, James; GRAINGER, Teresa; WRAY, David (ed.). Learning to Teach in the Primary School. London: Routledge, 2006. p. 209-252.

GROTH, John; PETERS, John. What Blocks Creativity? A Managerial Perspective. Creativity and Innovation Management, [S. l.], v. 8, n. 3, p. 179-187, 1999.

HAYES, Denis. Understanding creativity and its implications for schools. Improving Schools, [S. l.], v.7, n. 3, p. 279-286, 2004.

HENNESSEY, Beth A.; AMABILE, Teresa M. Creativity. Annual Reviews of Psychology, [S. l.], n. 61, p. 569-598, 2010.

HILAL, Huda Mohamad Hassan; HUSIN, Wan Nurul Izza Wan; ZAYED, Tareq Mohammad. Barriers to Creativity among Students of Selected Universities in Malaysia. International Journal of Applied Science and Technology, [S. l.], v. 3, n. 6, p. 51-61, 2013. Disponível em: http://www.ijastnet.com/journals/Vol_3_No_6_August_2013/7. pdf. Acesso em: 30 out. 2021.

HOFF, Eva. The creative place: The Impact of Different Environmental Factors on Creativity In: SHIU, Eric (ed.). Creativity Research: An Inter-Disciplinary and MultiDisciplinary Research Handbook. New York: Routledge, 2014. p. 103-126.

HUNTER, Samuel T.; BEDELL, Katrina E.; MUMFORD, Michael D. Climate for Creativity: A Quantitative Review. Creativity Research Journal, [S. l.], v. 19, n. 1, p. 69-90, 2007.

INTASAO, Nujaree; HAO, Ning. Beliefs About Creativity Influence Creative Performance: The Mediation Effects of Flexibility and Positive Affect. Frontiers in psychology, [S. l.], v. 9, p. 1-17, 2018. Disponível em: https:/www.frontiersin.org/ article/10.3389/fpsyg.2018.01810. Acesso em: 30 out. 2021.

JINDAL-SNAPE, Divya et al. The impact of creative learning environments on learners: A systematic literature review. Improving Schools, [S. l.], v. 16, n. 1, p. 21-31, 2013.

KABANDA, Patrick. The Creative Wealth of Nations: How the Performing Arts Can Advance Development and Human Progress. Washington, DC: World Bank Group, 2014.

KAMPYLIS, Panagiotis. Fostering creative thinking: the role of primary teachers. Jyvaskyla, FIN: University of Jyvaskyla, 2010.

KONSTANTINIDOU et al. Barriers and Inhibitors of Creativity in Physical Education. European Psychomotricity Journal, [S. l.], v. 7, n. 1, p. 17-31, 2015.

KOZBELT, Albert; BEGHETTO, Robert A.; RUNCO, Marko Andrew. Theories of Creativity In: STERNBERG, Robert J.; KAUFMAN, John C. (ed.). The Cambridge Handbook of Creativity. Cambridge: Cambridge University Press, 2010. p. 20-47. 
LUBART, Todd; ZENASNI, Frank; BARBOT, Baptiste. Creative Potential and Its Measurement. International Journal for Talent Development and Creativity, [S. l.], v. 1, n. 2, p. 41-51, 2013.

MARTIN, Lorna P. Inventory of Barriers to Creative Thought and Innovative Action. In: PFEIFFER, William (ed.). The 1990 Annual: Developing Human Resources. San Diego: University Associates, 1990. p. 138-141.

MORAIS, Maria Fatima; ALMEIDA, Leandro. I would be more creative if...: Are there perceived barriers to college students creative expression according to gender? Estudos de Psicologia, Campinas, v. 36, e180011, 2019. Disponível em: https:/www.scielo. $\mathrm{br} / \mathrm{j} /$ estpsi/a/DNkxTkCxHrN9DJFXXjavascript:void(0);f4qPfm/?lang=en\&format=p df. Acesso em: 30 out. 2021.

MORAIS, Maria Fatima et al. Perceptions of barriers to personal creativity: Validation of an inventory involving high education students. The European Journal of Social and Behavioral Sciences, [S. l.], v. 10, n. 3, p. 281-295, 2014. Disponível em: https://www. europeanpublisher.com/data/articles/131/5840/article_131_5840_pdf_100.pdf. Acesso em: 30 out. 2021.

NORDIN, Norshidah; MALIK, Melissa. Undergraduates' Barriers to Creative Thought and Innovative in a New Millennial Era. Procedia - Social and Behavioral Sciences, $[S$. l.], n. 201, p. 93-101, 2015.

OECD. Education at a Glance 2011 OECD Indicators: OECD Indicators. Paris: OECD Publishing, 2011.

PALETZ, Susannah B. Project Management in Innovative Teams. In: MUMFORD, Michael D. (ed.). Handbook of Organizational Creativity. London: Academic Press, 2012. p. 421-455.

PARAMITHA, Anggia; INDARTI, Nurul. Impact of the Environment Support on Creativity: Assessing the Mediating Role of Intrinsic Motivation. Procedia - Social and Behavioral Sciences, [S. l.], n. 115, p. 102-114, 2014.

PARASKEVOPOULOS, Ioannis; PARASKEVOPOULOU, Polyxeni. $\Delta \eta \mu 10 v \rho \gamma 1 \kappa \eta$

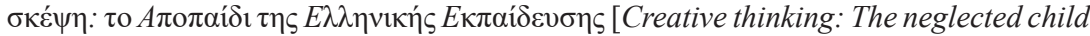
of Greek education]. Athens: Koralli, 2009.

PARKHURST, Howard B. Confusion, Lack of Consensus, and the Definition of Creativity as a Construct. The Journal of Creative Behavior, [S. l.], v. 33, n. 1, p. 1-21, 1999. Disponível em: https://onlinelibrary.wiley.com/doi/10.1002/j.2162-6057.1999. tb01035.x. Acesso em: 30 out. 2021.

PAULHUS, Delroy L.; VAZIRE, Simine. The Self-Report Method. In: ROBINS, R. W.; FRALEY, R. C., et al (ed.). Handbook of Research Methods in Personality Psychology. 
New York: Guilford Press, 2007. p. 224-239. Disponível em: https:/www2.psych. ubc.ca/ dpaulhus/research/SDR/downloads/CHAPTERS/2008\%20Handbook\%20 Research\%20Methods/paulhus-vazire\%2007\%20chap.pdf. Acesso em: 30 out. 2021.

PETER-SZARKA, Szilvia. Creative Climate as a Means to Promote Creativity in the Classroom. Electronic Journal of Research in Educational Psychology, [S. l.], v. 10, n. 3, p. 1011-1034, 2012.

PINTRICH, Paul R.; DE GROOT, Elisabeth. Motivational and Self-Regulated Learning Components of Classroom Academic Performance. Journal of Educational Psychology, [S. l.], v. 82, n. 1, p. 33-40, 1990.

PLUCKER, Jonathan A.; MAKEL, Matthew C. Assessment of Creativity. In: STERNBERG, Robert J.; KAUFMAN, James C. (ed.). The Cambridge Handbook of Creativity. Cambridge: Cambridge University Press, 2010. p. 48-73.

PRABHU, Veena; SUTTON, Charlotte; SAUSER, William. Creativity and Certain Personality Traits: Understanding the Mediating Effect of Intrinsic Motivation. Creativity Research Journal, [S. l.], v. 20, n. 1, p. 53-66, 2008.

PUCCIO, Gerard; GRIVAS, Chris. Examining the Relationship between Personality Traits and Creativity Styles. Creativity and Innovation Management, [S. l.], v. 18, n. 4, p. 247-255, 2009.

ROBINSON, Ken; ARONICA, Lou. Creative Schools: The Grassroots Revolution That's Transforming Education. London: Penguin Publishing Group, 2016.

SADI, Muhammad Asad; AL-DUBAISI, Ali H. Barriers to organizational creativity: The marketing executives' perspective in Saudi Arabia. Journal of Management Development, [S. l.], v. 27, n. 6, p. 574-599, 2008.

SHAHEEN, Robina. Creativity and Education. Creative Education, [S. l.], v. 1, n. 3, p. 166-169, 2010.

SIMONTON, Dean Keith. Creativity: Cognitive, personal, developmental, and social aspects. American Psychologist, [S. l.], v. 55, n. 1, p. 151-158, 2000.

STERNBERG, Robert J. The Nature of Creativity. Creativity Research Journal, [S. l.], v. 18 , n. 1, p. $87-98,2006$.

VALENZUELA VIANNA, Claudia R.; ALENCAR, Eunice M. L. Creativity and Barriers to its Expression in Online Education Courses. Gifted Education International, [S. l.], v. 21, n. 1, p. 54-62, 2006.

VERNON, Philip E. The Nature-Nurture Problem in Creativity. In: GLOVER, John A.; RONNING, Royce R.; REYNOLDS, Cecil R. (ed.). Handbook of Creativity. New York, NY: Springer Science \& Business Media LLC, 1989. p. 93-110. 


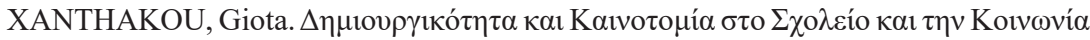
[Creativity and innovation in school and society]. Athens: Diadrasi, 2012.

ZBAINOS, Dimitrios; BELOYIANNI, Vasiliki. Creative Ideation and Motivated Strategies for Learning of Academically Talented Students in Greek Secondary School. Gifted and Talented International, [S. l.], v. 33, n. 1-2, p. 3-14, 2018.

ZIEGLER, Albert; VIALLE, Wilhelmina. The Tower of Creativity. In: INTERNATIONAL CONFERENCE ON THE CULTIVATION AND EDUCATION OF CREATIVITY AND INNOVATION, 2009, Xi'an, China. Papers [...]. Xi'an, China: Institute of Psychology of Chinese Academy of Sciences, 2009. p. 28-40. Disponível em: https://ro.uow.edu.au/ edupapers/997/. Acesso em: 30 out. 2021.

ZIEGLER, Albert. The impact of beliefs on intelligence, achievement and creativity. In: ZIEGLER, Albert et al. (ed.). Gifted Education as a Lifelong Challenge. Essays in Honour of Franz J. Monks. Berlin: LIT, 2012. p. 337-350.

Texto recebido em 06/09/2021.

Texto aprovado em 23/08/2021. 